\title{
Axillary Reconstruction Using a Pedicled Thoracodorsal Artery Perforator Flap Including Latissimus Dorsi Muscle Strip
}

\author{
Jin An Cha (D), Sung Ho Yoon (1D) \\ Department of Plastic and Reconstructive Surgery, Haeundae Paik Hospital, Inje University College of Medicine, Busan, Korea
}

\begin{abstract}
For axillary reconstruction, most reconstructive surgeons use the latissimus dorsi flap because of its usefulness and reliability. However, the latissimus dorsi musculocutaneous flap poses complications such as donor site morbidity and poor aesthetic results. Moreover, the thoracodorsal artery perforator (TDAP) flap, without muscle, presents difficulties for the delicate procedure of dissecting the perforators separately. Therefore, we propose the use of a pedicled TDAP flap including a latissimus dorsi muscle strip for axillary reconstruction, as it is believed to be easier and safer to perform.
\end{abstract}

Keywords: Perforator flap; Axilla; Surgical flaps; Reconstructive surgical procedures

\section{Introduction}

Covering large soft tissue defects when managing conditions such as postburn contractures, chronic hidradenitis suppurativa, or ectopic carcinoma in the axillary region can be challenging. In traditional reconstruction, split-thickness or full-thickness skin grafts and flap reconstruction are used for coverage. However, these techniques have shortcomings, such as contractures, donor site morbidity, aesthetically poor appearance, and the sacrifice of major blood vessels [1]. To overcome these limitations, perforator-based fasciocutaneous flaps have been considered as a reliable and suitable surgical option. However, identifying the perforators and dissecting them meticulously is difficult; this procedure is challenging to learn and requires a long operative time. Therefore, for axillary reconstruction, we propose the use of a pedicled thoracodorsal artery perforator (TDAP) flap including a latissimus dorsi muscle strip. We present our experience with two patients who required coverage for axillary defects to describe the technique and utility of pedicled TDAP flap with muscle strip for coverage of the axillary region.

\section{Case}

\section{Patients}

The first patient had a burn scar contracture in his left axilla and could not move his arm freely. The second patient had been diagnosed with chronic hidradenitis suppurativa, a skin disease, in his right axilla. Both patients were admitted to the plastic and

\section{Case Report}

Received: February 26, 2019

Revised: July 3, 2019

Accepted: July 4, 2019

Corresponding author: Sung Ho Yoon, M.D.

Department of Plastic and Reconstructive Surgery, Haeundae Paik Hospital, Inje University College of Medicine, 875 Haeundaero, Haeundae-gu, Busan 48108, Korea Tel: +82-51-797-2380

Fax: +82-51-797-2386

E-mail: Chaja1225@gmail.com

This is an Open Access article distributed under the terms of the Creative Commons Attribution Non-Commercial License (https://creativecommons.org/licenses/by-nc/4.0/) which permits unrestricted non-commercial use, distribution, and reproduction in any medium, provided the original work is properly cited.

C 2020 Korean Wound Management Society 
reconstructive surgery department for axillary problems and required additional coverage for the soft tissue defects that developed after treatment. Both patients underwent reconstructive surgery using a pedicled TDAP flap including a latissimus dorsi muscle strip. The skin paddle was designed based on the needs of the defect site. The study was performed in accordance with the principles of the Declaration of Helsinki. Written informed consents were obtained.

\section{Surgical technique}

The perforators originate from the descending branch of the thoracodorsal artery, which is mainly located between 8 and $13 \mathrm{~cm}$ (mean, $10.8 \mathrm{~cm}$ ) distal to the axillary fold and within 0 to $5 \mathrm{~cm}$ (mean, $2.8 \mathrm{~cm}$ ) of the anterior border of the latissimus dorsi muscle [2]. Based on this vascular anatomy, a Doppler probe is used to identify the descending branch of the thoracodorsal artery; therefore, landmarking can be performed preoperatively.

The patient is placed in a lateral decubitus position with the relevant upper arm abducted. After palpating and marking the anterior border of the latissimus dorsi muscle, the anterior end of the skin paddle is designed about $1 \mathrm{~cm}$ in front of the anterior edge of the latissimus dorsi muscle to ensure that the flap contains the main perforators. The design of skin paddle is determined according to the shape and size of the defect site and possibility of primary closure. The main perforator usually lies along and approximately $2 \mathrm{~cm}$ posterior to the anterior border of the latissimus dorsi muscle, about $8 \mathrm{~cm}$ inferior to the inferior border of the axilla [3]. Other perforators mostly lie in a row along the descending branch of thoracodorsal artery.

After the skin paddle design is created over the latissimus dorsi muscle, the anteroinferior skin incision is made and is extended downward until the anterior border of the latissimus dorsi muscle is identified. Dissection proceeds posteriorly and upward along the suprafascial layer until the perforators are detected.

When a suitable perforator complex is found, the dissection is stopped. Suprafascial dissection in the superior and inferior direction is limited to $2 \mathrm{~cm}$ from the main perforator, because further dissection can injure the cutaneous branches of the thoracodorsal artery.

To isolate the underlying thoracodorsal pedicle, the anterior border of the latissimus dorsi muscle is elevated and the dissection of the muscle is continued to find the entry point of the perforator.

When the main pedicle is identified, the muscle flap is lifted and isolated to incorporate the pedicle and any of its perforators into the flap. The pedicle width of the latissimus dorsi muscle harvested can range from 3 to $4 \mathrm{~cm}$. Subsequently, the posterior incision of the skin paddle is continued until the suprafascial layer is reached; likewise, on the other side, dissection is limited to $2 \mathrm{~cm}$ from the perforator. Finally, the flap's skin paddle, including the latissimus dorsi muscle strip where the descending branch of the thoracodorsal artery follows, is separated completely from the remainder of the muscle. Muscle dissection is then continued in the cephalad direction until the flap, including the muscle strip, is sufficiently tension-free. The flap's skin paddle can be transposed to cover the recipient site, and the remaining latissimus dorsi muscle is repaired to minimize the dead space and prevent possible complications such as late serous formation. A negative pressure suction drain is inserted and primary repair is conducted layer by layer.

\section{Cases}

Case 1

A 56-year-old male patient visited our plastic surgery department with the chief complaint of postburn scar contracture in his axillary region. He had a restricted range of motion at the axilla as a result of a flame burn that had occurred 20 years earlier; he had not previously undergone any surgical intervention to release the contracture. He could not raise and stretch his arm freely and the range of motion was about $60^{\circ}$. He needed release of the scar contractures, which would have resulted in a relatively large soft-tissue defect in this region. Therefore, we decided to release the contracture and reconstruct the defect using a TDAP flap including a latissimus dorsi muscle strip.

Preoperative perforator mapping was performed using a Doppler probe, with the patient in a lateral decubitus position. To adequately expose the fields of the axilla and latissimus dorsi muscle, we suspended and fixed the elbow and forearm safely in the caudal direction. We then palpated and marked the anterior border of the latissimus dorsi muscle. A Doppler probe was used to identify the descending branch of the thoracodorsal artery. After scar tissue was removed, the contracting bands were completely released. The wound size was $5 \mathrm{~cm} \times 8$ $\mathrm{cm}$ and a quadrilateral-shaped skin paddle sized $6 \mathrm{~cm} \times 9 \mathrm{~cm}$ was designed around the point indicated by the Doppler signal. The skin incision was made and continued down to the latissimus fascia. The dissection then began suprafascially at the anterior border of the flap and was continued carefully. The anterior border of the latissimus dorsi muscle was lifted to 
identify the descending branch of the thoracodorsal artery. The muscle was split vertically along its natural muscle fiber orientation posterior to the pedicle, which is $1 \mathrm{~cm}$ medial to the descending branch of the thoracodorsal artery. The skin flap, including the muscle strip, was transposed to the axillary defect without any tension and donor site was closed primarily in usual fashion with a single suction drain (Fig. 1).

The patient was successfully provided with complete release of the scar contracture and reconstruction of the axillary defect. Postoperatively, the scar contracture of his axilla healed completely with a cosmetically acceptable result; full extension was achieved after a gradual increase in daily activities over 2 months (Fig. 2).

\section{Case 2}

An obese 18-year-old man had chronic hidradenitis suppurativa in his right axilla. Multiple drainages of abscesses combined with medical management had been performed for over 3 years but had failed to provide any lasting relief. The condition relapsed numerous times along with infection, causing difficulty in arm abduction. The patient needed surgical excision of the affected tissue in his axilla and coverage of the defect was inevitable.

After placing the patient in the right lateral decubitus posi- tion with the upper arm at $90^{\circ}$ abduction and $90^{\circ}$ flexion at the elbow, anatomical points were marked and the perforators were identified with care. We then performed complete resection of the involved tissue, leaving a $9 \mathrm{~cm} \times 10 \mathrm{~cm}$-sized defect in the right axilla. The upper third of the defect was closed primarily by undermining the subcutaneous layer, but the remaining $6 \mathrm{~cm} \times 6 \mathrm{~cm}$ defect could not be resolved. We therefore planned the pedicled TDAP flap including latissimus dorsi muscle strip for the remaining defect. The design and harvest technique of the flap was almost identical to that performed in case 1 . A $7 \mathrm{~cm} \times 10 \mathrm{~cm}$ skin paddle with the latissimus dorsi muscle strip was designed and then advanced to the defect site without any tension. The patient was provided with a successful axillary reconstruction with a safe and aesthetically satisfactory result. At 3 weeks postoperatively, the flap had healed and he had functional use of the shoulder. There was no recurrence of the hidradenitis suppurativa after surgery (Fig. 3).

The method with the pedicled TDAP flap including the latissimus dorsi muscle strip turned out to be quick and reliable. The operations took about 150 and 180 minutes, respectively. The axillary defect was covered without any tension, and all the flaps used in the reconstruction healed uneventfully. No complications such as partial flap loss, wound dehiscence, se-
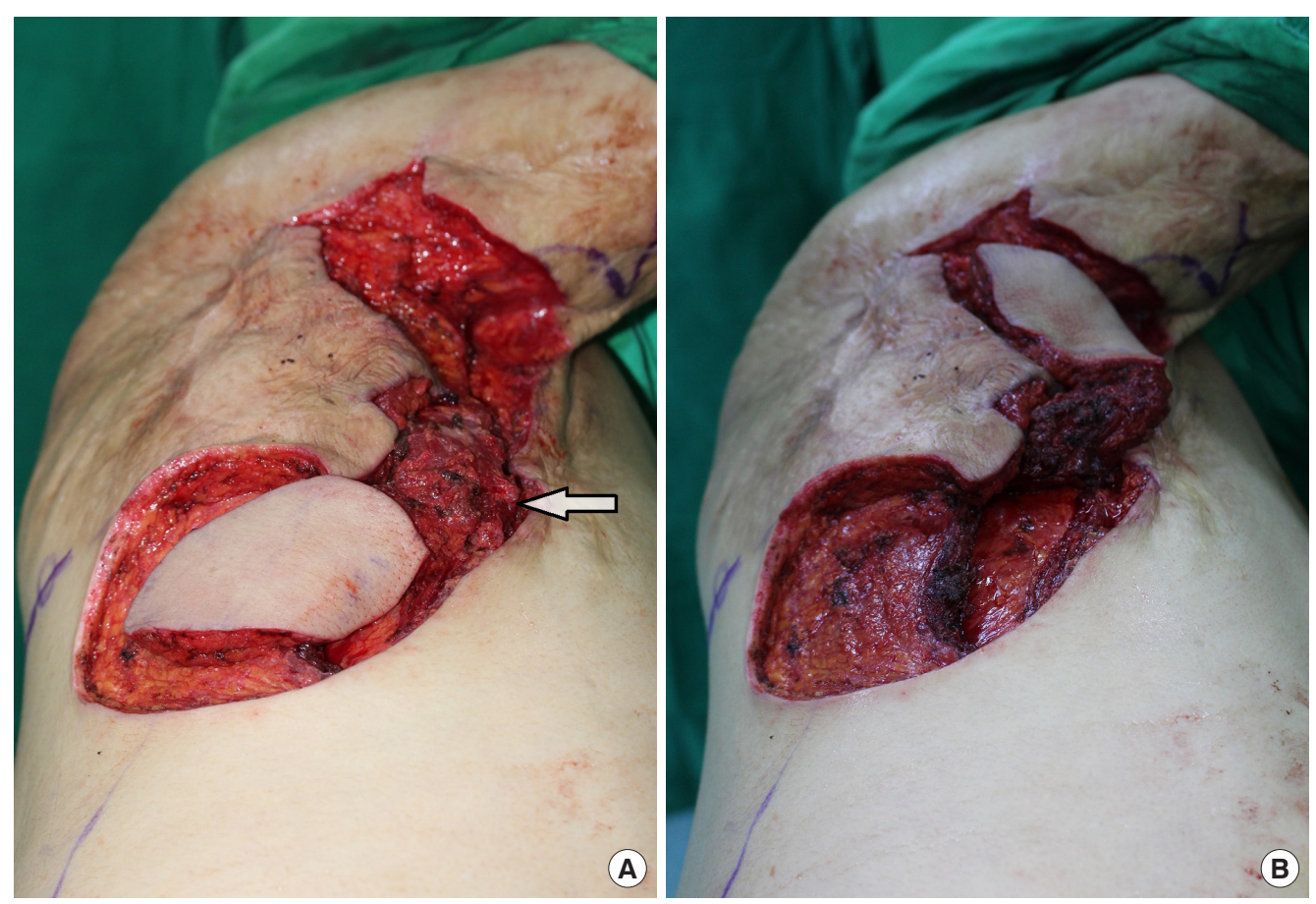

Fig. 1. Intraoperative view of case 1. (A) Intraoperative view shows the muscle strip and elevated flap (arrow). (B) The flap inset for the defect site. 
Cha JA et al.

Pedicled TDAP flap including LD muscle strip
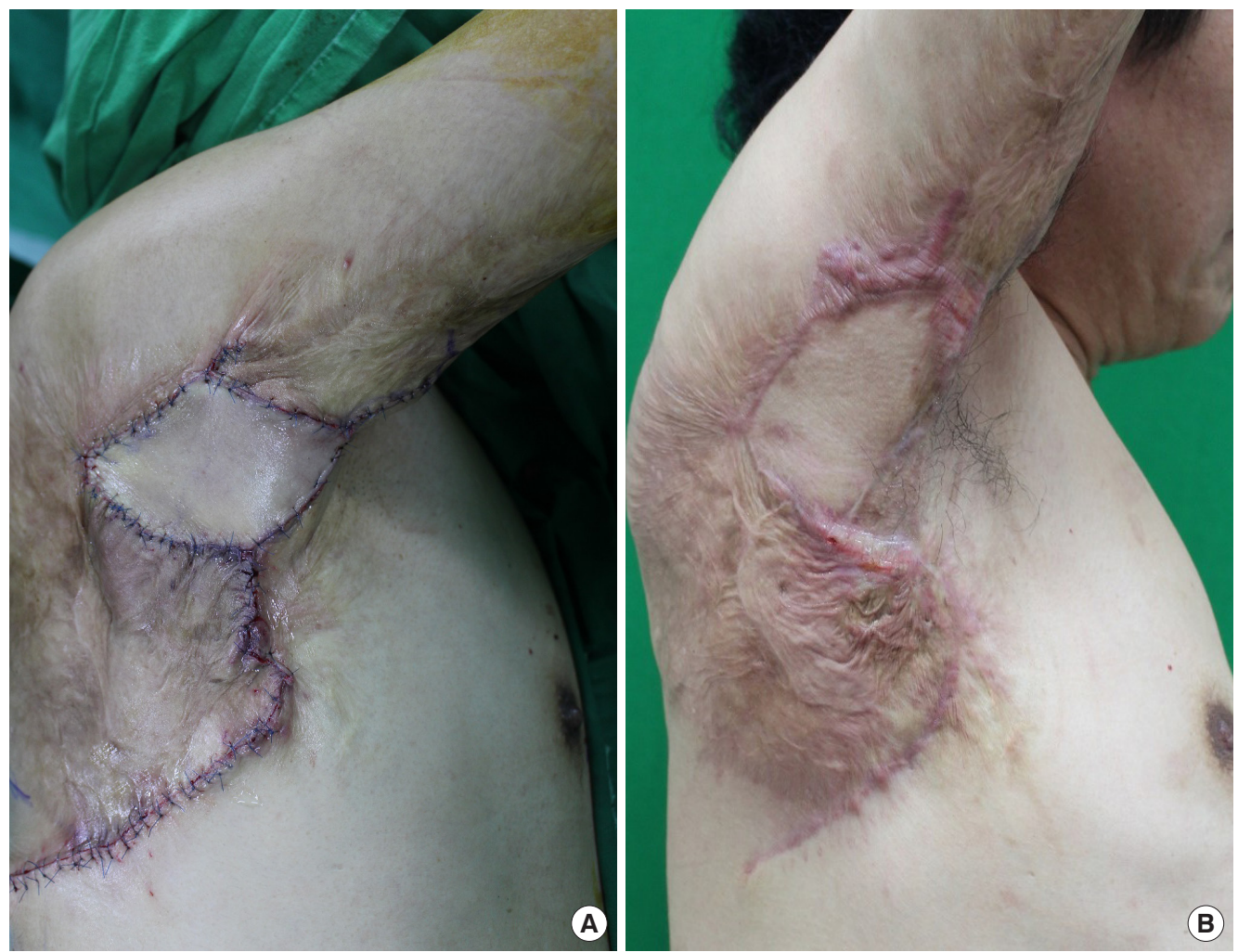

Fig. 2. Postoperative view of case 1. (A) Postoperative view after flap closure. (B) Postoperative view at 3 months follow-up.
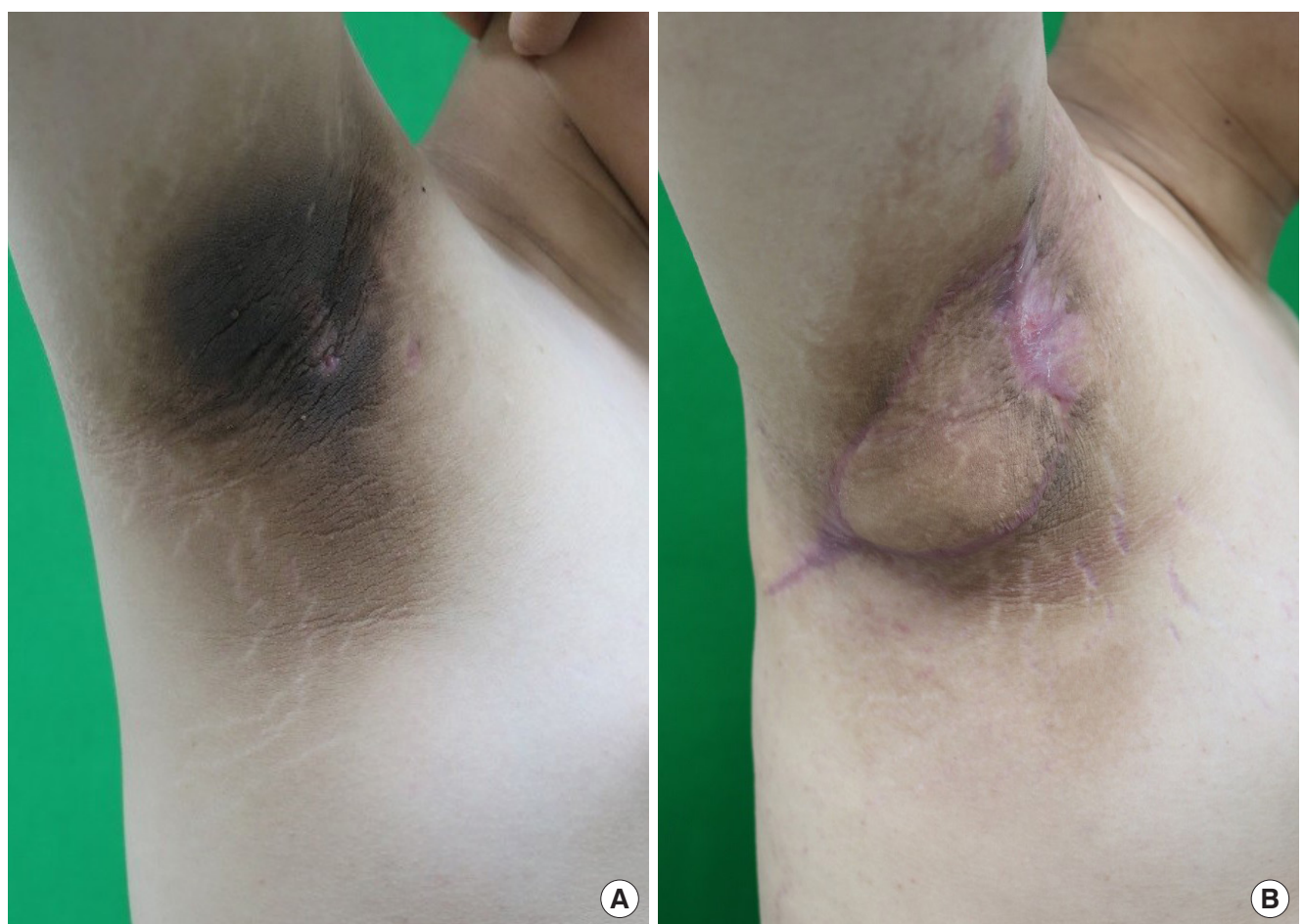

Fig. 3. Preoperative and postoperative view of case 2. (A) The patient had chronic hidradenitis suppurativa in his right axilla. (B) Eighteen months postoperative view of axillar reconstruction with pedicled thoracodorsal artery perforator flap including latissimus dorsi muscle strip technique. 
roma, or hematoma occurred postoperatively. All the flaps remained safe and aesthetically satisfactory, and all donor sites healed with favorable results.

\section{Discussion}

The TDAP flap was first described by Angrigiani et al. in 1995 [3]. It was first introduced and accepted for the purpose of lessening donor site disability caused by harvest of latissimus dorsi musculocutaneous flap. The TDAP flap became a popular candidate in reconstructing soft-tissue defects such as those in the breasts, axillae, upper limbs, upper back, and neck [4]. This perforator flap is used widely because of the following attributes: reliable anatomy, the flap's capacity to thin without compromising its blood supply, the possibility of obtaining a long pedicle, the pliability of the flap, the potential of preparing a chimeric flap on the subscapular vascular system, and the lack of significant donor site morbidity [4-6].

Despite its advantages, using the TDAP flap is quite challenging. The dissection of perforator and pedicle demands meticulous skill. In case of an injury to the perforator, there would be a high risk of poor perfusion, leading to failure.

Furthermore, there is a high risk for perforator rupture or avulsion when the diameter of the perforator is less than 0.5 $\mathrm{mm}$. In such situations, the use of a pedicled TDAP flap with muscle strip can be helpful, because it enhances the viability by preserving the muscle around the perforators as well as saving the operation time by making it possible to skip intramuscular dissection $[7,8]$.

Including this small portion of the muscle while maintaining direct visualization of the perforators reduces the risk of breakage during the repositioning of the flap. In addition, unlike the usual perforator flap, this pedicled perforator flap with muscle strip does not rely only on the main perforator, but also includes various muscular branches. This means that a more reliable flap can be achieved, and with some minor perforators, the flap can be lengthened along the axis of the descending branch of the thoracodorsal artery, further enhancing the utility of the technique.

The minimal morbidity of the donor site is another great advantage of the technique, particularly when comparing to the use of the conventional latissimus dorsi musculocutaneous flap. Although the dissection and flap elevation procedure may kill minor nerve branches, in most cases, the latissimus dorsi preserves muscle strength and the full range of shoulder movements after surgery [9].
As the pedicle is harvested in strip form, thickness of the flap is minimally modified. The shape of the back is preserved and there is no need for further flap debulking.

This method can be even safer and more useful when used for axillary reconstruction than when it is used for reconstruction of the breast, neck, and upper limb, because a long pedicle is not needed for coverage of the axillary defect [10]. It is easy to perform in terms of dissecting the flap, as well as safe because it preserves the muscle strip, including the main pedicle. This concept allows surgeons to save time and effort required in surgical procedures to elevate a flap.

In conclusion, this study shows the safety and utility of using the pedicled TDAP flap including a latissimus dorsi muscle strip in axillary reconstruction. This flap has the advantages of less functional limitation of the donor site, greater modifiability of the location of the skin paddle, and an aesthetically favorable result. Therefore, we recommend the pedicled TDAP flap including a latissimus dorsi muscle strip as a reliable method for axillary reconstruction.

\section{Conflict of interest}

No potential conflicts of interest relevant to this article are reported.

\section{ORCID iDs}

Jin An Cha https://orcid.org/0000-0001-5112-9697

Sung Ho Yoon https://orcid.org/0000-0002-9978-2828

\section{References}

1. Adams WP Jr, Lipschitz AH, Ansari M, et al. Functional donor site morbidity following latissimus dorsi muscle flap transfer. Ann Plast Surg 2004;53:6-11.

2. Hamdi M, Van Landuyt K, Hijjawi JB, et al. Surgical technique in pedicled thoracodorsal artery perforator flaps: a clinical experience with 99 patients. Plast Reconstr Surg 2008; 121:1632-41.

3. Angrigiani C, Grilli D, Siebert J. Latissimus dorsi musculocutaneous flap without muscle. Plast Reconstr Surg 1995;96: 1608-14.

4. Uygur F, Sever C, Tuncer S, et al. Reconstruction of postburn antebrachial contractures using pedicled thoracodorsal artery perforator flaps. Plast Reconstr Surg 2009;123:154452. 
5. Jandali S, Mirzabeigi MN, Fosnot J, et al. Thoracodorsal artery perforator flaps and muscle-sparing latissimus dorsi myocutaneous flaps for the treatment of axillary hidradenitis. Ann Plast Surg 2012;69:371-5.

6. Rehman N, Kannan RY, Hassan S, et al. Thoracodorsal artery perforator (TAP) type I V-Y advancement flap in axillary hidradenitis suppurativa. Br J Plast Surg 2005;58:441-4.

7. Zhu L, Mohan AT, Saint-Cyr M. A simple approach to harvest of the pedicled descending branch muscle-sparing latissimus dorsi flap. J Plast Reconstr Aesthet Surg 2015;68: e179-81.
8. Saint-Cyr M, Nagarkar P, Schaverien M, et al. The pedicled descending branch muscle-sparing latissimus dorsi flap for breast reconstruction. Plast Reconstr Surg 2009;123:13-24.

9. Hamdi M, Decorte T, Demuynck M, et al. Shoulder function after harvesting a thoracodorsal artery perforator flap. Plast Reconstr Surg 2008;122:1111-7.

10. Schwabegger AH, Harpf C, Rainer C. Muscle-sparing latissimus dorsi myocutaneous flap with maintenance of muscle innervation, function, and aesthetic appearance of the donor site. Plast Reconstr Surg 2003;111:1407-11. 